\title{
Tratamiento farmacológico para COVID-19 en protocolos latinoamericanos: una revisión narrativa de la eficacia y seguridad
}

\author{
Pharmacological treatment for COVID-19 in latin american \\ protocols: A narrative review of the effectiveness and safety
}

\author{
Pamela Alejandra \\ Escalante Saavedral,* (iD) \\ Martín Cañás" \\ Dulce María Calvo Barbado"'I (iD) \\ Liliana Barajas Esparzalv iD \\ Mariana Caffarattiv iD \\ Noelia Speranza Mourine ${ }^{\text {vI }}$ iD \\ Carlos Alberto \\ Fuentes Martínez ${ }^{\mathrm{VII}}$
}

José Julián López Gutiérrez ${ }^{\mathrm{VIII}}$ (iD

1 Centro Brasileiro de Informação sobre Medicamentos, Conselho Federal de Farmácia, Brasília, DF, Brasil

" CIMEFF Área de Farmacología, Federación Médica de la Provincia de Buenos Aires (FEMEBA), La Plata, Argentina

III Asesora independiente, Barcelona, España

Iv Instituto de Ciencias de la Salud, Universidad Autónoma del Estado de Hidalgo, Pachuca de Soto, Hidalgo, México

$\checkmark$ Centro de Información de Medicamentos, Facultad de Ciencias Químicas, Universidad Nacional de Córdoba, Córdoba, Argentina

VI Departamento de Farmacología y Terapéutica, Facultad de Medicina, Universidad de la República, Montevideo, Uruguay

VII Centro de Información de Medicamentos CIME, Acción Internacional por la Salud (AIS Nicaragua), Matagalpa, Nicaragua

VIII Departamento de Farmacia, Facultad de Ciencias, Universidad Nacional de Colombia, Bogotá, Colombia

* E-mail: saavedra.pamela.alejandra@ gmail.com

Recibido: 07 ago 2020

Aceptado: 13 ago 2020

\section{RESUMEN}

Introducción: La pandemia causada por el SARS-Cov-2 ha puesto a la comunidad científica mundial en ritmo acelerado de investigación y busca por un tratamiento efectivo para COVID-19. Objetivo: Identificar y evaluar medicamentos en protocolos latinoamericanos de tratamiento farmacológico para el COVID-19. Método: Se analiza la evidencia y resultados de mega ensayo disponibles hasta la fecha sobre los medicamentos más frecuentes. Resultados: Los medicamentos más frecuentes en protocolos nacionales son hidroxicloroquina, lopinavir/ritonavir y remdesivir. Ninguno de los medicamentos que recopilan mayor cantidad de datos provenientes de estudios clínicos, a excepción de la dexametasona en un subgrupo reducido de pacientes, mostró, hasta el momento, diferencias significativas en la mortalidad. Conclusiones: La situación emergente de la COVID-19 ha determinado la toma de decisiones apresuradas y controversiales con base en estudios cuestionables y/o de baja calidad. Esto pone de relieve el carácter provisorio de la información y la posibilidad de generar cambios a medida que se dispongan de más resultados. La autorización anticipada de medicamentos expone un problema conocido. A pesar de que la agilidad regulatoria es necesaria en este momento, la velocidad no debe sobreponerse a los patrones básicos éticos y de confianza en la evidencia.

PALABRAS CLAVE: COVID-19; Protocolos Clínicos; Medicamentos; Medicina Basada en Evidencia; Latinoamérica

\begin{abstract}
Introduction: The pandemic caused by SARS-CoV-2 has put the global scientific community in an accelerated pace of research for an effective treatment for COVID-19. Objective: To identify and evaluate drugs in Latin American protocols of pharmacological treatment for COVID-19. Method: The evidence and mega trial results available to date on the most frequent medications are analyzed. Results: The most common drugs in national protocols are hydroxychloroquine, lopinavir/ritonavir, and remdesivir. None of the drugs that collect the most data from clinical studies, with the except for dexamethasone in a small subgroup of patients, so far showed significant differences in mortality. Conclusions: The emerging situation of COVID-19 has determined hasty and controversial decision-making based on questionable and/or low-quality studies. This highlights the provisional nature of the information and the possibility of generating changes as more results become available. Advance medication authorization exposes a known problem. Although regulatory agility is required at this time, speed should not overlap with basic ethical standards and trust in evidence.
\end{abstract}

KEYWORDS: COVID-19; Clinical protocols; Medicaments; Evidence-Based Medicine; Latin America 


\section{INTRODUCCIÓN}

El nuevo coronavirus o SARS-CoV-2 causa manifestaciones clínicas que pueden presentarse como cuadros leves, moderados 0 graves, lo que incluye neumonía, síndrome de distrés respiratorio agudo (SDRA), sepsis y shock séptico. La mayoría de los casos notificados debutan con cuadros leves ${ }^{1}$.

En América Latina, los países oficializaron protocolos de tratamiento farmacológico en los que incluyen medicamentos para diferentes condiciones clínicas de los pacientes (gravedad) y en diferentes niveles de atención ${ }^{2}$. Sin embargo, varios de estos protocolos dejan la decisión sobre el tratamiento farmacológico a utilizar en cada paciente a criterio médico, es decir, es el médico quien debe evaluar la relación beneficio/riesgo y decidir con base en la evidencia disponible y su experiencia personal.

Entre los medicamentos incluidos se destaca hidroxicloroquina (HCQ), cloroquina (CQ) (dos antipalúdicos con efectos inmunomoduladores también usados para tratar afecciones autoinmunes como lupus eritematoso sistémico y artritis reumatoide) ${ }^{3}$, remdesivir (un profármaco análogo nucleotídico con actividad antiviral por inhibición de la ARN polimerasa dependiente de ARN 96\% idéntica entre síndrome respiratorio de Oriente Medio - MERS, síndrome respiratorio agudo severo - SARS y COVID-19)², lopinavir/ritonavir (LPV/r, combinación de antivirales utilizados en el tratamiento del virus de inmunodeficiencia humana - VIH) ${ }^{2}$ y más recientemente dexametasona (un corticosteroide que pudiera ser útil en la reducción de las complicaciones del síndrome de dificultad respiratoria aguda SDRA) en las formas graves de la enfermedad causada por el SARS-CoV- $2^{4}$.

Además de las medidas de soporte para aquellos pacientes que requieren hospitalización, no existe actualmente evidencia procedente de ensayos clínicos controlados de calidad, publicados en revistas con revisión por pares, para recomendar un tratamiento específico para el coronavirus SARS-CoV-2 ${ }^{1,5,6}$.

A continuación, se analiza la evidencia disponible hasta la fecha de elaboración de este artículo, sobre los medicamentos más frecuentemente incluidos en los protocolos de tratamiento farmacológico de COVID-19 en Latinoamérica.

\section{MÉTODO}

Se realizó una revisión de los protocolos nacionales para COVID-19 de países latinoamericanos los cuales fueron suministrados por los centros de información pertenecientes a la Red de Centros de Información de Medicamentos de Latinoamérica y el Caribe (RED CIMLAC), así como de las guías de práctica clínica del Instituto Nacional de Salud (NIH por su sigla en inglés) de los Estados Unidos. Se incluyeron convenientemente aquellos medicamentos que representaran un mecanismo de acción diferente, fármacos más referenciados en los protocolos latinoamericanos y ensayos clínicos internacionales.

De esta manera, se incluyó CQ e HCQ como representante de los antiparasitarios, remdesivir y LPV/r como representantes del grupo de antivirales y dexametasona como representante de corticosteroides.
Para la identificación de resultados de eficacia y seguridad de los medicamentos seleccionados entre abril y junio de 2020, se revisaron las bases de datos de MEDLINE (motor de búsqueda PubMed), Epistemonikos, EMBASE, Literatura Latinoamericana y del Caribe en Ciencias de la Salud (LILACS) y Cochrane Library, en las cuales se identificaron estudios clínicos con los fármacos seleccionados. La estrategia y palabras claves empleadas en PubMed fueron las siguientes: (Therapy/Broad[filter]) AND (-nombre del fármaco-); (Therapy/Broad[filter]) AND (-nombre del fármaco- AND COVID 19); (Therapy/Broad[filter]) AND (-nombre del fármaco- AND SARS-CoV-2); (Therapy/Broad[filter]) AND (-nombre del fármaco- AND coronavirus); ("-nombre del fármaco-" [Supplementary Concept]) AND “COVID-19" [Supplementary Concept]; (Medical Genetics[filter]) AND (-nombre del fármaco); (Therapy/Broad[filter]) AND (-nombre del fármacoAND efficacy); ("Treatment Outcome"[Mesh]) AND “-nombre del fármaco-" [Supplementary Concept]); (“-nombre del fármaco-” [Supplementary Concept]) AND "Drug-Related Side Effects and Adverse Reactions"[Mesh]); (Therapy/Broad[filter]) AND (-nombre del fármaco- AND adverse events).

Adicionalmente, se realizó una revisión no sistemática de repositorios institucionales de agencias sanitarias como la Food and Drug Administration (FDA) y Agencia Española de Medicamentos y Productos Sanitarios (AEMPS), y del proyecto Observatorio de Medicamentos de Alto Impacto Financiero (DIME) ${ }^{7}$, de la Biblioteca de la Universidad Pablo de Olavide de España ${ }^{8}$, del Instituto de Salud Carlos III del Ministerio de Ciencia e Innovación español ${ }^{9}$ y la base Clinical Trials del Instituto Nacional de Salud de los Estados Unidos ${ }^{10}$.

Se revisaron los resultados preliminares del estudio Randomised Evaluation of COVID-19 Therapy (RECOVERY), un ensayo clínico aleatorizado (ECA) multicéntrico abierto con seis ramas de tratamiento: $\mathrm{LPV} / \mathrm{r}, \mathrm{HCQ}$, dexametasona, azitromicina, plasma de convalecientes y tocilizumab ${ }^{11}$.

Se incluyeron para su análisis estudios observacionales analíticos y ensayos clínicos, además de resultados preliminares y series de casos de los fármacos seleccionados.

\section{RESULTADOS}

\section{Cloroquina e hidroxicloroquina}

Según los resultados de Wang et al. ${ }^{12}$, Yao et al. ${ }^{13}$ y Mc Intosh ${ }^{14}$, CQ e HCQ inhiben la replicación del SARS-CoV-2 in vitro aunque HCQ parece tener una actividad antiviral más potente. Basándose en estos estudios in vitro, la Comisión Nacional de Salud de China fue la primera a incluir el uso de CQ en sus guías de tratamiento ${ }^{14,15}$.

En la Tabla 1, ocho ECA y varios estudios observacionales de CQ e HCQ. El estudio RECOVERY es el que aporta mayor evidencia y concluye que no se observaron diferencias en la mortalidad cuando los pacientes son tratados con HCQ. La evidencia de los ensayos clínicos hasta el momento no ha demostrado beneficios, ya sea como tratamiento o como profilaxis. 
En cuanto a los eventos adversos que ocurrieron con el uso de CQ e HCQ, varios estudios clínicos y observacionales, suman evidencia de que este tratamiento se asocia con efectos adversos cardíacos, como la prolongación del intervalo QT. Los principales reportes se describen en la Tabla 1.

A continuación, se resumen las recomendaciones y advertencias que sociedades científicas, organizaciones y agencias sanitarias han realizado en los últimos meses, para reducir el riesgo de arritmias en pacientes en tratamiento de COVID-19 con CQ o HCQ $28,32,33,34$ :

i. Monitorizar el intervalo electrocardiográfico/QT.

ii. Corregir la hipopotasemia (a valores superiores a $4 \mathrm{mEq} / \mathrm{L}$ ) e hipomagnesemia (a valores superiores a $2 \mathrm{mg} / \mathrm{dL}$ ).

iii. Utilizar con precaución en pacientes con enfermedad cardíaca y renal. Se debe realizar una evaluación inicial de estos factores y un monitoreo continuo.

iv. No combinar con otros medicamentos que comparten riesgo de prolongar el intervalo QT del electrocardiograma (ECG). El riesgo aumenta con dosis altas de HCQ y cuando se administra con otros medicamentos que también comparten este posible riesgo, como azitromicina.

v. Usar HCQ sólo en el ámbito hospitalario o en el contexto de un ensayo clínico, debido a sus potenciales efectos adversos cardíacos graves y la necesidad de corregir alteraciones electrolíticas, además de monitorear y evaluar las funciones hepática y renal.

vi. En el caso que un paciente sea tratado en forma extrahospitalaria, informar a los pacientes sobre el riesgo de alteraciones del ritmo cardiaco, su sintomatología y la necesidad de consultar al médico en caso de que éstos aparezcan.

\section{Remdesivir}

Según la base de datos Clinical Trials del NHI, al momento de escribir este artículo hay al menos 15 ECA en curso con tratamiento estándar en pacientes con COVID-19 moderada y grave (nueve de ellos en fase 3$)^{2}$, todos sin resultados publicados todavía. En la Tabla 2 se describen los resultados publicados de los estudios más relevantes y los eventos adversos relatados.

Con base en el estudio de Beigel et al. ${ }^{36}$, la FDA otorgó la autorización de uso de emergencia a remdesivir en pacientes hospitalizados con COVID-19 grave ${ }^{40}$. Este es el que aporta mayor evidencia hasta el momento; sus resultados preliminares mostraron que el tiempo promedio de recuperación fue de 11 días en el grupo remdesivir, comparado con 15 días en el grupo placebo. Sin embargo, no se observaron diferencias estadísticamente significativas en la mortalidad.

Además, los resultados del estudio de Goldman et al..$^{39}$ con remdesivir y un artículo editorial acompañante advierte que en los momentos actuales de suministros limitados de remdesivir, para pacientes en las fases iniciales de la enfermedad grave, se debe dar prioridad a un tratamiento de cinco días ${ }^{41}$.

\section{Lopinavir/ritonavir}

LPV/r es una combinación de antivirales para el VIH, donde lopinavir es el agente activo que inhibe la actividad proteasa del coronavirus, mientras que ritonavir aumenta la vida media de lopinavir. Esta asociación mostró actividad in vitro en SARS-CoV y MERS-CoV por lo cual se postuló su uso como parte del tratamiento de la COVID-1942. En la Tabla 3 se describen los resultados más relevantes. El estudio RECOVERY, que aporta mayor evidencia hasta el momento, no mostró beneficio sobre la progresión de la enfermedad a necesidad de ventilación mecánica ni sobre la duración de estancia hospitalaria.

Además, sobre la seguridad se relata que LPV/r causa efectos adversos gastrointestinales (diarrea, náusea, vómitos) ${ }^{44,46}$ y también infección respiratoria alta ${ }^{46}$, dislipidemia, disglucemia, prolongación del intervalo QT y tiene el potencial de interactuar con una gran cantidad de medicamentos ${ }^{47}$.

\section{Dexametasona}

Sobre dexametasona, los estudios encontrados están descritos en la Tabla 4.

La mayor evidencia hasta ahora proviene del ensayo clínico RECOVERY ${ }^{49}$, los resultados preliminares muestran que, en pacientes hospitalizados en estado crítico, la dexametasona redujo las muertes en $1 / 3$ en pacientes ventilados y en $1 / 5$ en otros pacientes que recibieron oxígeno solamente. No se encontró evidencia de beneficio en pacientes hospitalizados que no requerían oxígeno y los resultados son consistentes con posibles daños en este grupo.

Se destaca que los autores del ECA multicéntrico español concluyeron que la administración temprana de dexametasona podría reducir la duración de la ventilación mecánica y la mortalidad general en pacientes con SDRA de moderado a severo establecido, sin diferencia significativa en la proporción de eventos adversos en ambos grupos ${ }^{48}$.

Por otra parte, una revisión sistemática a partir de estudios observacionales y una pequeña cohorte con datos en pacientes con COVID-19, publicada en mayo 2020, señala que los corticosteroides pueden reducir la mortalidad en pacientes con COVID-19 y SDRA, pero que en los pacientes con COVID-19 grave sin SDRA, la evidencia sobre el beneficio era inconsistente y de muy baja calidad ${ }^{50}$.

Con base en esto, al producirse en las infecciones por SARS, MERS y COVID-19 inflamación y daño alveolar difuso con hemofagocitosis, se espera en consonancia con las fases clínicas de la enfermedad y la histopatología que el uso con corticoides (por ejemplo, dexametasona), podría tener un papel en la supresión de la inflamación pulmonar ${ }^{51,52,53}$.

El beneficio en pacientes graves que requieren oxígeno, observado en el ensayo RECOVERY recientemente publicado, sumado a su perfil de seguridad conocido, el bajo costo y la gran accesibilidad, posiblemente conduzcan a su adopción como parte de los protocolos en este grupo de pacientes ${ }^{49}$. 
Tabla 1. Ensayos clínicos y observacionales con hidroxicloroquina y cloroquina en COVID-19 (principales resultados de eficacia y seguridad).

\begin{tabular}{llc}
\hline Autores & Descripción & Principales resultados \\
\hline Estudios clínicos aleatorizados & &
\end{tabular}

Treinta pacientes hospitalizados con infección moderada sin

comorbilidades significativas (edad promedio de 50 años, $70 \%$

Chen et al. ${ }^{16}$ hombres). El grupo tratamiento recibió HCQ $400 \mathrm{mg} / \mathrm{dí}$ y el grupo control tratamiento estándar durante cinco días. Todos los pacientes recibieron otros tratamientos en forma concomitante.

Chen et al. ${ }^{17}$

En 62 pacientes internados con neumonía, sin estado crítico (edad promedio de 45 años). Grupo tratamiento recibió $200 \mathrm{mg}$ cada 12 horas de HCQ durante cinco días además del tratamiento de sostén, y el grupo control sólo el tratamiento de sostén.

Resultados intermedios de ECA con 150 pacientes en relación $1: 1$ con COVID-19 leve a moderada y con edad promedio de 46 años. 75 pacientes fueron asignados a tratamiento con $800-1.200 \mathrm{mg} /$ día de HCQ más atención estándar y 75 pacientes solo a atención estándar. La duración media entre el inicio de los síntomas y el tratamiento con HCQ o atención estándar fue de 16 días. El 63\% de los pacientes en ambos grupos recibieron otros tratamientos con antivirales.

ECA con hallazgos preliminares sobre eficacia y seguridad de dos regímenes de CQ en 81 pacientes con COVID-19 severo. Se evidenció una mortalidad de 39,0\% (16/41 pacientes) en grupo de alta dosis versus $15,0 \%(6 / 40)$ en el grupo de baja dosis (log-rank, $-2.183 ; P=0,03$ ).

Borba et al. ${ }^{19}$

$\begin{array}{ll}\text { Boulware et al. }{ }^{20} & \text { HCQ Profilaxis post-exposición a COVID-19, } 441 \text { pacientes con } \\ \text { BCO } & \text { HCQ con placebo }(n=407) \text {; mediana de edad con }\end{array}$ HCQ 41 años, con placebo 40 años; 48,2\% hombres.

Chen et al. ${ }^{21}$

ECA, 48 pacientes de los cuales 18 con $\mathrm{HCQ}, 18$ con CQ y 12 con placebo; promedio de edad CQ de 45,2 años, HCQ de 45,7 años, placebo de 51,3 años; $46 \%$ hombres.
No se encontraron diferencias entre los grupos en la depuración viral nasofaríngea en el día 7 , ni en la duración de estancia hospitalaria o complicaciones.

RAM: No hubo diferencias en los eventos adversos entre los grupos. No hubo muertes.

HCQ acelera modestamente la desaparición de los síntomas (fiebre y tos) y la mejoría radiológica.

No presentan los resultados de la variable principal del protocolo original: depuración viral y tiempo de recuperación de células $\mathrm{T}$.

El estudio presenta serias limitaciones y no fue revisado por pares, por lo que los resultados deben interpretarse con precaución.

La administración de HCQ no resultó en una probabilidad significativamente mayor de conversión negativa de la PCR a los 28 días de tratamiento.

RAM: Los eventos adversos fueron mayores en el grupo de HCQ ( $30 \%$ vs $8,8 \%$ ).

Concluyeron que no debe recomendarse $\mathrm{CQ}$ para pacientes críticos con COVID-19 debido a sus posibles riesgos de seguridad, especialmente cuando se toman simultáneamente con azitromicina y oseltamivir.

Limitaciones del estudio: falta de un grupo control con placebo, pequeño tamaño de muestra, ausencia de criterios de exclusión basados en el intervalo QTC al inicio del estudio. RAM: QTc elevado (11/73) por dosis altas. Mortalidad (16/41) por dosis altas.

Sin muertes en ninguno de los grupos; incidencia enfermedades compatibles con COVID-19 sin diferencias significativas entre los participantes que recibieron $\mathrm{HCQ}$ (49 de $414[11,8 \%]$ ) y los que recibieron placebo (58 de 407 $[14,3 \%])$; la diferencia absoluta fue $-2,4$ puntos porcentuales (intervalo de confianza del $95 \%-7,0$ a 2,2; $P=0,35$ ). RAM: Con HCQ los efectos no fueron graves, no se produjo arritmias. No hubo muertes.

El grupo CQ logró un tiempo más corto para la recuperación clínica (TRC) que el grupo control $(P=0,019)$. Hubo una tendencia hacia TRC reducido en el grupo HCQ $(P=0,049)$. El tiempo para alcanzar la negatividad del ARN viral fue significativamente más rápido en el grupo $C Q$ y el grupo HCQ que en el grupo control $(P=0,006$ y $P=0,010$, respectivamente). La mediana de días para alcanzar la negatividad de ARN en los grupos CQ, HCQ y control fue de 2,5 (RIC: 2,0-3,8) días; 2,0 (RIC: 2,0-3,5) días y 7,0 (RIC: 3,0-10,0) días, respectivamente. Los grupos CQ y HCQ también mostraron tendencias hacia la mejora en la duración de la hospitalización

ECA abierto. Rama de HCQ: 1.542 pacientes asignados a HCQ ( $2.000 \mathrm{mg}$ el primer día, $400 \mathrm{mg}$ a las 24 horas siguientes y $800 \mathrm{mg} /$ día por nueve días) y 3.132 pacientes asignados a la atención estándar. Variable principal: mortalidad. Variables secundarias: tiempo de estancia hospitalaria, necesidad de ventilación, necesidad de terapia de reemplazo renal y aparición de arritmias graves (incluida en una modificación del protocolo). No se excluyeron a los pacientes que recibían remdesivir.

ECA Profilaxis post-exposición a COVID-19. HCQ $800 \mathrm{mg}$ una vez, seguida de $600 \mathrm{mg}$ en 6 a 8 horas, luego $600 \mathrm{mg}$ diarios durante

Skipper et al. ${ }^{23} \quad 4$ días más, o placebo enmascarado.

HCQ 212 vs placebo 211. El 56\% (236 de 423) se inscribieron dentro de 1 día del inicio de los síntomas. y los hallazgos en la tomografía computarizada (TC) pulmonar. diferencias significativas en la variable primaria de mortalidad a los 28 días $(25,7 \%$ para HCQ versus $23,5 \%$ para la atención estándar $(H R=1,11 ; I C 95 \% 0,98-1,26 ; P=0,10)$. Tampoco

hubo evidencia de efectos beneficiosos sobre la duración de la estancia hospitalaria u otros resultados.

Sin diferencias en cambio en la severidad de los síntomas a los 14 días (diferencia en la severidad de los síntomas: relativa 12\%; absoluta $-0,27$ puntos [IC95\% $-0,61$ a 0,07 puntos]; $P=0,117$ ). recibieron HCQ tenían síntomas continuos en comparación con el $30 \%$ (59 de 194) que recibieron placebo $(P=0,21)$.
Resultados preliminares sólo de la rama de HCQ. No hubo A los 14 días, el $24 \%$ (49 de 201) de los participantes que 
Continuación

\begin{tabular}{|c|c|}
\hline Autores & Descripción \\
\hline \multicolumn{2}{|c|}{ Estudios observacionales } \\
\hline Gautret et al. ${ }^{24}$ & $\begin{array}{l}\text { En } 36 \text { pacientes con HCQ ( } 200 \text { mg tres veces al día durante } \\
\text { diez días). }\end{array}$ \\
\hline
\end{tabular}

En 1.438 pacientes hospitalizados con COVID-19 tratados con

Rosenberg et al. ${ }^{25} \mathrm{HCQ}$, azitromicina o ambos, en comparación con ninguno de los tratamientos.

Geleris et al.26 En 1.376 pacientes con COVID-19, la administración de HCQ, versus grupo control.

En 368 pacientes hombres, mediana de 69 años, tratados con Magagnoli et al. ${ }^{27}$ HCQ más el tratamiento de sostén. Las variables de desenlace fueron mortalidad y necesidad de ventilación mecánica.
El uso de HCQ se asoció con una tasa más alta de ARN de SARS-CoV-2 indetectable en muestras nasofaríngeas en el día 6 en comparación con ningún tratamiento específico $(70,0 \%$ versus $12,5 \%$ ). El uso de azitromicina en combinación con HCQ parece tener un beneficio adicional. Limitaciones: validez interna (no controlado, no aleatorizado, no enmascarado, con exclusión de pacientes sin adecuada justificación). La variable principal sin duración o relevancia clínica óptima lo que limita su validez externa.

No se asoció significativamente con diferencias en la mortalidad hospitalaria ni alteraciones en el ECG en el análisis ajustado (HR = 1,08 IC95\% 0,63-1,85; HR = 0,56 IC95\% 0,26-1,21 y HR = 1,35 IC95\% 0,76-2,40, respectivamente), en ninguno de los grupos de tratamiento.

No se asoció con un riesgo significativamente mayor o menor de intubación o muerte $(H R=1,04 ;$ IC95\% 0,82 a 1,32) en el análisis ajustado.

La tasa de mortalidad fue de $27,8 \%$ en aquellos tratados con $\mathrm{HCQ}$, de $22,1 \%$ en aquellos tratados con $\mathrm{HCQ}$ y azitromicina y de $11,4 \%$ en aquellos no tratados con HCQ. La tasa de ventilación fue $(13,3 \%),(6,9 \%)$ y $(14,1 \%)$, respectivamente. El uso de HCQ sola (pero no el uso de HCQ y azitromicina) se asoció con un aumento de la mortalidad general en comparación con el tratamiento de sostén, mientras que el uso de HCQ con o sin azitromicina no redujo el riesgo de ventilación mecánica.

Este resultado fue 20,5 versus $22,0 \%$, (16 versus 21 eventos, $R R=0,93 ;$ IC95\% 0,48-1,81). Ocho pacientes de los 84 que recibieron HCQ tuvieron modificaciones del ECG que requirieron interrupción del tratamiento.

En 181 pacientes que requerían oxígeno tratados con HCQ. Se comparó el uso de HCQ $600 \mathrm{mg}$ al día versus tratamiento Mahévas et al. ${ }^{28}$ estándar de sostén. Variable primaria: supervivencia sin necesidad de ingreso a cuidados intensivos a los siete días de evolución o muerte.

RAM: Modificaciones electrocardiográficas $(8 / 84)$, prolongación del intervalo QT corregido $>500 \mathrm{~ms}$ (1 paciente), bloqueo auriculoventricular de primer grado (1 paciente).

En 93\% de los pacientes se negativizó la PCR en el octavo día de tratamiento.

La mayor parte de los pacientes tenía cuadros leves a

Estudio retrospectivo, observacional y no controlado con 80 Gautret et al. ${ }^{29}$ pacientes que fueron tratados con HCQ (200 mg 3 veces al día durante diez días) y azitromicina (500 mg en el día 1, luego 250 $\mathrm{mg}$ diarios en los días 2-5).

Estudio observacional retrospectivo sin grupo control, con 1.061 pacientes SARS-CoV-2 positivos, independientemente de la presencia de síntomas. El 95,0\% de los pacientes tenían infección leve.

Million et al. ${ }^{30}$

Cohorte prospectiva de 11 pacientes hospitalizados (no

Molina et al. ${ }^{31}$ controlado). Todos los pacientes recibieron la misma dosis y duración de HCQ que el estudio de Gautret et al. ${ }^{24}$.

moderados, e incluso cuatro eran asintomáticos. Dado que además no hubo grupo control, impide conocer cuál hubiera sido su evolución espontánea y cuál puede ser la utilidad en pacientes con cuadros más graves.

Se observó mejoría clínica y depuración viral en diez días en $91,7 \%$ de los pacientes. Cinco continuaban internados al momento de la finalización del estudio.

RAM: Eventos adversos leves $(2,3 \%)$ de índole gastrointestinal y cutánea.

El régimen de $\mathrm{HCQ}$ y azitromicina no produjo un aclaramiento viral rápido ni proporcionó un beneficio clínico en pacientes graves.

Fuente: Autoría propia con datos de los estudios citados, 2020.

RAM: Reacciones adversas a medicamentos; ECA: Ensayo clínico aleatorizado; HCQ: Hidroxicloroquina; CQ: cloroquina; ECG: electrocardiograma.

\section{DISCUSIÓN}

Esta revisión consolida los datos provenientes de estudios clínicos disponibles sobre los medicamentos empleados en el COVID-19 más frecuentemente presentes en los protocolos de América Latina y de aquellos posicionados como candidatos a incluirse. El contexto latinoamericano con sus características asistenciales, sociales, económicas y la existencia de autoridades regulatorias con una menor fuerza en sus decisiones, complejiza la aplicación de la mejor evidencia disponible.

Los Centros de Información de la región en su formato de RED CIMLAC, han compilado esta información y han realizado recomendaciones a partir del análisis de los beneficios y riesgos de los medicamentos que se incluyen en las acciones terapéuticas contra el COVID-19 en la región. En ese sentido, se observa que la información proveniente de ensayos clínicos aleatorizados es limitada, incluyendo la investigación de diferentes desenlaces, lo que dificulta la comparación o agrupación y análisis estadístico que refuerce o refute los hallazgos.

Los medicamentos más frecuentemente presentes en protocolos nacionales y que recopilan mayor cantidad de datos provenientes de estudios clínicos son $\mathrm{HCQ}, \mathrm{LPV} / \mathrm{r}$ y remdesivir. Ninguno mostró, hasta el momento, diferencias significativas en la mortalidad.

En el caso de CQ e HCQ, la mayoría de los estudios previos a la diseminación de los resultados del estudio RECOVERY, fueron poco concluyentes y de calidad cuestionable. Al considerar la 
Tabla 2. Ensayos clínicos y observacionales con remdesivir en COVID-19 (principales resultados de eficacia y seguridad).

\begin{tabular}{|c|c|c|}
\hline Autor & Descripción & Principales resultados \\
\hline Wang et al. ${ }^{35}$ & $\begin{array}{l}\text { Ensayo clínico aleatorizado, doble ciego, controlado con placebo } \\
\text { y multicéntrico. } \\
237 \text { pacientes hospitalizados, con saturación de oxígeno < } 95 \% \text { o } \\
\mathrm{PaO} 2 / \mathrm{FiO} \leq 2300 \mathrm{mmHg} \text { y neumonía confirmada radiológicamente. } \\
\text { Grupo tratamiento: remdesivir }(\mathrm{n}=158 \text { ). Grupo control: placebo } \\
\text { (n=79). A ambos grupos se les permitió el uso concurrente de } \\
\text { corticosteroides, interferones y LPV/r. Variable principal: tiempo } \\
\text { hasta la mejora clínica, realizándose seguimiento durante } 28 \text { días. }\end{array}$ & $\begin{array}{l}\text { Finalizó prematuramente por dificultades de reclutamiento. } \\
\text { No se encontraron diferencias entre remdesivir y placebo en la } \\
\text { variable principal (HR }=1,23 \text {; IC } 95 \% \text { 0,87-1,75), ni en las variables } \\
\text { secundarias, incluyendo mortalidad. El tratamiento con remdesivir } \\
\text { no se asoció con beneficios estadísticamente significativos. } \\
\text { RAM: Hipotensión, náuseas, vómitos, diaforesis y temblores. }\end{array}$ \\
\hline Beigel et al. ${ }^{36}$ & $\begin{array}{l}\text { ECA, doble ciego, controlado con placebo con } 1.059 \text { pacientes } \\
\text { hospitalizados aleatorizados para recibir remdesivir ( } \mathrm{n}= \\
538 \text { ) o placebo }(\mathrm{n}=521) \text {. Pacientes tenían al menos uno de } \\
\text { los siguientes criterios de infección: infiltrados radiográficos } \\
\text { por estudio de imagen, saturación periférica de oxígeno } \\
\text { (SpO2 } \leq 94 \% \text { ) en aire ambiente, o requerimiento de oxígeno } \\
\text { suplementario, ventilación mecánica u oxigenación por } \\
\text { membrana extracorpórea. } \\
\text { El porcentaje de pacientes en ventilación mecánica en el } \\
\text { momento de la aleatorización fue de } 23,1 \% \text { en el grupo remdesivir } \\
\text { y de } 28,2 \% \text { en el grupo placebo. Excluidos pacientes con niveles } \\
\text { de ALT o AST > } 5 \text { veces el límite superior normal, con insuficiencia } \\
\text { renal, necesidad de hemodiálisis o hemofiltración. Duración media } \\
\text { de los síntomas antes del inicio de remdesivir fue de nueve días. } \\
\text { Variable principal: tiempo hasta la mejora clínica. }\end{array}$ & $\begin{array}{l}\text { El tiempo promedio de recuperación fue de } 11 \text { días en el grupo } \\
\text { de remdesivir, comparado con } 15 \text { días en el grupo placebo (HR = } \\
1,32 ; \text { IC } 95 \% 1,12 \text { - } 1,55 ; p<0,001 \text { ). También hubo una tendencia } \\
\text { hacia una menor mortalidad que no fue estadísticamente } \\
\text { significativa; } 7,1 \% \text { en el grupo de remdesivir versus } 11,9 \% \text { con } \\
\text { placebo (HR = 0,70; IC } 95 \% 0,47-1,04 \text { ). } \\
\text { Según autores, el porcentaje de mortalidad en el grupo } \\
\text { de remdesivir, es alto a pesar del tratamiento, por lo que } \\
\text { probablemente como estrategia futura se pueda evaluar la } \\
\text { combinación de remdesivir con otros tratamientos antivirales } \\
\text { para mejorar los resultados en los pacientes con COVID-19. } \\
\text { Remdesivir mostró una mejoría en el tiempo de recuperación de } \\
\text { cuatro días respecto del tratamiento de soporte. Este dato, a } \\
\text { pesar de que podría considerarse beneficioso por algunos en el } \\
\text { contexto de una pandemia, presenta escasa relevancia clínica. } \\
\text { RAM: Se informaron eventos adversos graves en } 21 \% \text {, anemia o } \\
\text { disminución de la hemoglobina, lesión renal aguda, disminución } \\
\text { de la tasa de filtración glomerular estimada, aumento de la } \\
\text { creatinina en sangre, pirexia, hiperglucemia y aumento de nos } \\
\text { niveles ALT, AST o ambas. }\end{array}$ \\
\hline
\end{tabular}

Serie de casos, en que se incluyeron 61 pacientes hospitalizados con saturación de oxígeno del $94 \%$ o menos, que iniciaron el Grein et al $^{37}$ tratamiento con remdesivir (uso compasivo). De éstos, ocho fueron excluidos por pérdida de datos, analizando finalmente 53 pacientes. La duración media de los síntomas antes del inicio de remdesivir fue de 12 días.

En estudio (preimpresión) que incluyó 35 pacientes hospitalizados en un hospital en Milán (Italia), con requerimientos de oxígeno o ventilador mecánico, a los que se les realizó seguimiento durante el uso compasivo de este

Antinori et al. ${ }^{38}$ fármaco. Fueron excluidos los pacientes con niveles de ALT o AST mayores de cinco veces el límite superior normal y depuración de creatinina $<30 \mathrm{~mL} / \mathrm{min}$. El resultado primario del estudio fue la mejoría clínica de los pacientes en el día 10 y 28.

Durante una mediana de seguimiento de 18 días, dos tercios de los casos tratados con remdesivir mostró una mejoría clínica; pero la ausencia de controles impide estimar la eficacia real. RAM: Hipotensión (8\%).

Diez pacientes tuvieron mejoría clínica al décimo día y 22 al vigésimo octavo; 14 fallecieron.

RAM: ocho pacientes abandonaron el estudio por eventos

adversos: cuatro por falla renal (tres fallecieron), tres por aumento de transaminasas hepáticas y uno por rash maculopapular severo.

En estudio abierto incluyó a 397 pacientes hospitalizados que fueron asignados al azar a 5 versus 10 días de remdesivir (dosis de carga de $200 \mathrm{mg}$ en el día 1 , seguido de $100 \mathrm{mg}$ al día). El estudio comparó la mejoría clínica de los pacientes de ambos

Goldman et al. ${ }^{39}$ grupos. Se incluyeron en el estudio pacientes hospitalizados con infección confirmada por SARS-CoV-2, saturación de oxígeno del $94 \%$ o menos mientras respiraban el aire ambiente y evidencia radiológica de neumonía.

Fuente: Autoría propia con datos de los estudios citados, 2020.

RAM: Reacciones adversas a medicamentos; ALT: alanina aminotransferasa AST: aspartato aminotransferasa.

Los resultados no mostraron una diferencia significativa entre los dos regímenes, en pacientes con COVID-19 grave que no requieren ventilación mecánica. evidencia disponible, se observa claramente que estos fármacos no son efectivos en el tratamiento de COVID-19, además de que su uso expone los pacientes a eventos cardíacos preocupantes. Así, CQ e HCQ no deberían ser recomendadas en protocolos nacionales o institucionales en los países en que se estén utilizando para tratar COVID-19.

Un hecho que merece la atención y que ha puesto a la comunicación científica en guardia, fue la gran repercusión el estudio de Mehra et al. ${ }^{54}$, que, a la fecha de elaboración de este artículo, es el que habría incorporado la muestra más amplia de pacientes y sugería una mayor mortalidad asociada al uso de HCQ y CQ en el contexto de la infección por COVID-19. Sin embargo, tras surgir importantes dudas con respecto a la integridad de la base de datos, una de las revistas científicas más renombradas,
The Lancet, emitió en primer lugar una nota de preocupación y posteriormente la retractación del estudio, a la que se sumaron tres de los cuatro autores.

Esta revisión evidencia que los estudios, tanto de remdesivir como de LPV/r, no han mostrado ningún beneficio sobre la mortalidad en COVID-19. El caso del remdesivir muestra otra de las aristas complicadas del manejo de la presente epidemia. La autorización anticipada (uso en emergencia) de remdesivir por el FDA pone de relieve la influencia comercial que emerge y presiona la regulación de los medicamentos en situación de pandemia ${ }^{55}$. La interrupción del ensayo, el cambio de la variable final, desde mortalidad hasta tiempo de recuperación sintomática y la diseminación de los resultados inicialmente a través de comunicados de prensa, expone este antiguo problema y la vulnerabilidad de 
Tabla 3. Ensayos clínicos y observacionales con lopinavir/ritonavir en COVID-19 (principales resultados de eficacia y seguridad).

\begin{tabular}{|c|c|c|}
\hline Autores & Descripción & Principales resultados \\
\hline Cao et al. ${ }^{43}$ & $\begin{array}{l}\text { Ensayo abierto, aleatorizado y abierto en } 199 \text { pacientes } \\
\text { hospitalizados con infección grave (dificultades para mantener } \\
\text { la saturación de } \mathrm{O}_{2}>94 \% \text { ). Fueron } 99 \text { pacientes para recibir LPV/r } \\
\text { (LPV } 400 \mathrm{mg} / 100 \mathrm{mg} \text { por vía oral/12 días) y } 100 \text { para recibir } \\
\text { atención estándar, durante } 14 \text { días. } \\
\text { El resultado primario fue la mejoría clínica en } 2 \text { puntos de } \\
\text { una escala ordinal de } 7 \text { puntos, o al alta hospitalaria, lo que } \\
\text { ocurriera primero. }\end{array}$ & $\begin{array}{l}\text { No fueron encontradas diferencias entre los dos grupos en el } \\
\text { resultado primario. La depuración viral no fue diferente entre } \\
\text { los grupos. La mortalidad fue menor en el brazo de tratamiento, } \\
\text { pero no fue estadísticamente significativa. } \\
\text { En } 14 \% \text { de los pacientes del grupo de tratamiento se tuvo que } \\
\text { suspender la intervención debido a efectos adversos como } \\
\text { intolerancia gastrointestinal y anormalidades de laboratorio. }\end{array}$ \\
\hline Hung et al. ${ }^{44}$ & $\begin{array}{l}\text { Ensayo abierto, aleatorizado, de fase } 2,127 \text { pacientes con } \\
\text { enfermedad leve a moderada fueron asignados aleatoriamente } \\
\text { ( } 2: 1) \text {, a una triple combinación de LPV/r }(400 \mathrm{mg}+100 \mathrm{mg} \text {, } \\
\text { a cada } 12 \text { horas, } 14 \text { días), ribavirina ( } 400 \mathrm{mg} \text { cada } 12 \text { horas) } \\
\text { e interferón beta- } 1 \mathrm{~b} \text { (tres dosis de } 8 \mathrm{millones} \text { de Ul, en días } \\
\text { alternos) o al grupo control LPV/r ( } 400 \mathrm{mg}+100 \mathrm{mg} \text {, a cada } 12 \\
\text { horas, } 14 \text { días). } \\
\text { La variable de desenlace principal fue el tiempo hasta obtener } \\
\text { un hisopado nasofaríngeo negativo por RT-PCR terapia. Sólo } \\
\text { recibieron interferón si se presentaron dentro de los siete días } \\
\text { posteriores al inicio de los síntomas. }\end{array}$ & $\begin{array}{l}\text { La variable principal fue significativamente más corta en el } \\
\text { grupo de terapia de combinación ( } 7 \text { versus } 12 \text { días; HR }=4,37 \text {; } \\
\text { IC95\% } 1,86-10,24) \text {. El grupo de combinación también tuvo } \\
\text { mejores resultados clínicos, incluido un tiempo más corto } \\
\text { para el alivio de los síntomas ( } 4 \text { versus } 8 \text { días) y una estancia } \\
\text { hospitalaria mediana más corta ( } 9 \text { versus } 15 \text { días). }\end{array}$ \\
\hline RECOVERY ${ }^{45}$ & $\begin{array}{l}\text { ECA abierto. Rama de LPV/r } 4.972 \text { pacientes. Fueron } 1.596 \\
\text { pacientes asignados LPV/r y } 3.376 \text { pacientes asignados a } \\
\text { tratamiento de sostén. }\end{array}$ & $\begin{array}{l}\text { Del total de pacientes analizados }(n=4.972), 26 \% \text { no requirió } \\
\text { ningún soporte respiratorio, } 70 \% \text { requirieron oxígeno suplementario } \\
\text { y } 4 \% \text { recibieron ventilación mecánica al ingresar al estudio. } \\
\text { No hubo efecto sobre la mortalidad a } 28 \text { días }(\mathrm{LPV} / \mathrm{r}=353 \\
(22,1 \%) \text {; control }=719(21,3 \%), \mathrm{RR}=1,04 \mathrm{IC} 95 \% 0,91-1,18) \text {. } \\
\text { Sin beneficio sobre la progresión de la enfermedad a necesidad } \\
\text { de ventilación mecánica y tampoco en la duración de estancia } \\
\text { hospitalaria (no se presentan medidas de asociación para } \\
\text { estos desenlaces). }\end{array}$ \\
\hline
\end{tabular}

Fuente: Autoría propia con datos de los estudios citados, 2020.

LPV/r: lopinavir/ritonavir; RT-PCR: transcripción reversa seguida de reacción en cadena de polimerasas.

Tabla 4. Ensayos clínicos aleatorizados con dexametasona en COVID-19 (principales resultados de eficacia y seguridad).

\begin{tabular}{|c|c|c|}
\hline Autores & Descripción & Principales resultados \\
\hline Villar et al. ${ }^{48}$ & $\begin{array}{l}\text { ECA que incluyó a } 277 \text { pacientes con SDRA en } 17 \text { unidades } \\
\text { de cuidados intensivos en hospitales universitarios de toda } \\
\text { España. Se aleatorizaron } 139 \text { en el grupo de dexametasona } \\
\text { y } 138 \text { en el grupo control. La variable principal de estudio } \\
\text { fue el número de días que el paciente permanecía vivo y sin } \\
\text { ventilador, desde el día de su aleatorización al día } 28 \text {. }\end{array}$ & $\begin{array}{l}\text { Para los pacientes del grupo de dexametasona, el número medio } \\
\text { de días fue mayor que el del grupo control (diferencia entre grupos } \\
4,8 \text { días [IC95\% } 2,57 \text { a } 7,03] ; p<0,0001 \text { ). La segunda variable } \\
\text { medida correspondió a todas las causas de muerte a los } 60 \text { días de } \\
\text { aleatorización. Se observó que } 29 \text { ( } 21 \% \text { ) pacientes en el grupo de } \\
\text { dexametasona y } 50(36 \%) \text { pacientes del grupo control habían muerto } \\
\text { (diferencia entre grupos }-15,3 \% \text { [IC } 95 \%-25,9 \text { a }-4,9] ; p=0,0047 \text { ). } \\
\text { RAM: Hiperglucemia, nuevas infecciones, barotraumas. No hubo } \\
\text { diferencias en los eventos adversos entre los grupos. }\end{array}$ \\
\hline RECOVERY ${ }^{49}$ & $\begin{array}{l}\text { La rama de dexametasona incluyó a } 2.104 \text { pacientes asignados } \\
\text { al azar a dexametasona } 6 \mathrm{mg} \text { una vez al día (por vía oral o IV) } \\
\text { durante diez días, comparados con } 4.321 \text { pacientes asignados } \\
\text { al azar al tratamiento estándar. }\end{array}$ & $\begin{array}{l}\text { La dexametasona redujo las muertes en } 1 / 3 \text { en pacientes ventilados } \\
\text { (relación de frecuencia } 0,65 ; \text { IC } 95 \% 0,48 \text { a } 0,88 ; p=0,0003 \text { ), y en } \\
1 / 5 \text { en otros pacientes que recibieron oxígeno solamente (relación } \\
\text { de frecuencia } 0,80 ; \text { IC } 95 \% 0,67 \text { a } 0,96 ; p=0,0021 \text { ). No se encontró } \\
\text { ningún beneficio en aquellos pacientes que no necesitaban asistencia } \\
\text { respiratoria (razón de frecuencia } 1,22 ; \text { IC } 95 \% 0,86 \text { a } 1,75 ; p=0,14 \text { ), } \\
\text { y se menciona que los resultados son consistentes con posibles } \\
\text { daños en este grupo. En general, la dexametasona redujo la tasa de } \\
\text { mortalidad a } 28 \text { días en } 17 \%, p=0,0007 \text {. }\end{array}$ \\
\hline
\end{tabular}

Fuente: Autoría propia con datos de los estudios citados, 2020.

SDRA: síndrome de distrés respiratorio agudo.

las instituciones ministeriales y de sanidad frente a la influencia comercial. A pesar de que la agilidad regulatoria es necesaria en este momento, la velocidad no debe sobreponerse a los patrones básicos éticos y de confianza en la evidencia ${ }^{56,57}$.

Por otro lado, de acuerdo a los datos del estudio RECOVERY, el uso de corticoides en el reducido grupo de pacientes que cumplan con los criterios de dicho estudio hace que sea una de las terapias consideradas como prometedoras.

El debate sobre las Guías Nacionales de tratamiento es importante, porque el hecho de que un medicamento esté incluido puede incentivar la automedicación y la búsqueda de esos medicamentos de manera indiscriminada por parte de la población para usos fuera de los detallados en los protocolos, por ejemplo, usos profilácticos, además de generar una falsa ilusión de prevención y protección en un momento en que el uso de máscaras, el lavado de manos y el distanciamiento social son imprescindibles. Por otro lado, el consumo masivo de medicamentos en uso off label como los utilizados en COVID-19, sea con o sin prescripción u orientación de un profesional de salud, puede llevar a un aumento de eventos adversos graves como los mencionados anteriormente.

Este trabajo, realizado de manera independiente, puede ser útil para apoyar a las instituciones de regulación y ministerios de salud en la definición de protocolos de atención con conductas 
basadas en evidencias robustas e imparciales. Los centros de información de medicamentos así como los comités de farmacología y terapéutica son aliados importantes en estos contextos de información precoz, parcial, de calidad variable y en cantidades excesivas. Además, los protocolos requieren constante revisión para poder acompasar los cambios de la evidencia y su comunicación adecuada por canales oficiales.

Lo anterior, principalmente porque la pandemia nos ha enseñado cuán importantes son los ensayos clínicos aleatorizados para apoyar decisiones en salud pública. El desafío fue, y sigue siendo, conciliar la urgencia de actuar con la generación de nuevos conocimientos y su aplicabilidad. Cualquier uso experimental de fármacos debería realizarse en un marco de investigación, con un protocolo definido, y una rigurosa recopilación e interpretación de los datos, y dentro del marco de un ensayo clínico ${ }^{57}$.

Entre las enseñanzas deseables que la comunidad científica puede extraer de este caso particular se destacan la necesidad de transparencia integral en los datos que sustentan las publicaciones, el riesgo de acelerar el proceso de publicación, la cautela a guardar en relación a las expectativas depositadas en la tecnología big data o la necesaria corresponsabilidad de los autores de un artículo con las bases de datos de sus propios estudios ${ }^{54,58}$. La información disponible en forma masiva, por las plataformas de pre publicación y de resultados preliminares, amplificada por los medios de comunicación, redes sociales y líderes políticos, han exagerado la magnitud y factibilidad de aplicación de los resultados, generando mucha presión sobre los profesionales de la salud y decisores sanitarios ${ }^{59}$.

En ese sentido, es necesario separar los datos provenientes de la investigación in vitro, los datos de series de casos reducidas, además de reforzar la necesidad de evitar los sesgos, dentro de lo posible en el escenario de la investigación procedente de la propia práctica clínica y de estar al tanto de los datos de seguridad de las nuevas terapéuticas propuestas con su inclusión al elaborar recomendaciones en las guías clínicas. Así, es necesario que los ECA contemplen análisis internos, protocolos adaptativos y otras estrategias en este sentido para dar más robustez y seguridad a la información que se obtiene de ellos, con revisores externos que monitoricen continuamente su evolución ${ }^{60}$. Finalmente, la necesidad de brindar al profesional sanitario de la región de fuentes de información más fiables y válidas.

La influencia negativa de las redes sociales y los medios sobre la población en general hace necesario elaborar propuestas de información entendibles dirigidas para hacer que el mensaje basado en evidencias llegue a este tipo de público con vistas a disminuir la automedicación.
El establecimiento de programas de farmacovigilancia intensiva que supervisen la seguridad en el uso de medicamentos off label y en pacientes muy heterogéneos se contempla como una necesidad no suficientemente reflejada en los hechos. Contar con un repositorio multicéntrico de todos los eventos adversos que se han producido por los tratamientos utilizados, con registros de cualquier país, permitiría realizar análisis profundos y representativos de los mismos, y puede ser una estrategia de seguridad que merece ser evaluada por las autoridades sanitarias de la región.

Finalmente, en el escenario de la COVID-19, se han sumado nuevos actores de la esfera política, que ha impulsado e implementado estrategias sin considerar la opinión técnica de las instituciones sanitarias; esto ha complejizado más la toma de decisiones por autoridades regulatorias que aparentan no ser totalmente independientes. En este particular, el dotar a las autoridades regulatorias de información científica de calidad debe ser uno de los objetivos esenciales de aquellos que la producen en la región, donde los centros de información de medicamentos juegan un papel protagónico para alcanzar este propósito.

\section{CONCLUSIONES}

Ninguno de los medicamentos que recopilan mayor cantidad de datos provenientes de estudios clínicos, a excepción de la dexametasona en un subgrupo reducido de pacientes con COVID-19 grave, mostró, hasta el momento, diferencias significativas en la mortalidad.

A la fecha, no se han publicado estudios que comparen los distintos tratamientos. Existen en curso varios estudios clínicos, los cuales aportarán mayor evidencia y que han de ser tomados en consideración para el manejo terapéutico de la enfermedad una vez analizados críticamente.

La evidencia disponible en la actualidad no permite hacer recomendaciones sobre el tratamiento específico de COVID-19.

La situación emergente de la COVID-19 ha determinado la toma de decisiones apresuradas y controversiales con base en estudios cuestionables y/o de baja calidad. La evidencia proveniente de estudios clínicos tiene limitaciones importantes, se investigan desenlaces diferentes, y con frecuencia no permite comparación o agrupación y análisis estadístico que refuerce los hallazgos. Esto pone de relieve el carácter provisorio de la información y la posibilidad de generar cambios a medida que se dispongan de más resultados.

La autorización anticipada de medicamentos expone un problema conocido. A pesar de que la agilidad regulatoria es necesaria en este momento, la velocidad no debe sobreponerse a los patrones básicos éticos y de confianza en la evidencia.

\section{REFERENCIAS}

1. Ministerio de Sanidad, Consumo y Bienestar Social (ES). Documento técnico: manejo clínico del COVID-19: atención hospitalaria. Madrid: Ministerio de Sanidad, Consumo y Bienestar Social; 2020[acceso 18 jun
2020]. Disponible en: https://www.mscbs.gob.es/ profesionales/saludPublica/ccayes/alertasActual/ nCovChina/documentos/Protocolo_manejo_clinico_ah_ COVID-19.pdf 
2. Red de Centros de Información de Medicamentos de Latinoamerica y el Caribe - CIMLAC. Tratamiento farmacológico para COVID-19: ¿cuál es la evidencia existente? Washington: Red de Centros de Información de Medicamentos de Latinoamerica y el Caribe; 2020[acceso 15 mayo 2020]. Disponible en: https://web2.redcimlac.org/ images/files/tratamientos_farmacologicos_final.pdf

3. Cennimo D, Windle M, Bronze M. Coronavirus disease 2019 (COVID-19) treatment \& management. Medscape. 23 mar 2020[acceso 24 mar 2020]. Disponible en: https://emedicine. medscape.com/article/2500114-treatment

4. Randomised Evaluation of COVID-19 Therapy - RECOVERY. Low-cost dexamethasone reduces death by up to one third in hospitalised patients with severe respiratory complications of COVID-19. London: University of Oxford; 2020[acceso 16 jun 2020]. Disponible en: https: / /www. recoverytrial.net/news/low-cost-dexamethasone-reducesdeath-by-up-toone-third-in-hospitalised-patients-withsevere-respiratory-complications-of-COVID-19

5. Kalil AC. Treating COVID-19: off-label drug use, compassionate use, and randomized clinical trials during pandemics. JAMA. 2020;323(19):1897-8. https://doi.org/10.1001/jama.2020.4742

6. Paumgartten FJR, Delgado IF, Pitta LR, Oliveira ACAX. Drug repurposing clinical trials in the search for life-saving COVID-10 therapies: research targets and methodological and ethical issues. Vigil Sanit Debate. 2020;8(2):39-53. https://doi.org/10.22239/2317-269x.0159

7. Decisiones Informadas en Medicamentos - DIME. Observatorio de medicamentos de alto impacto financiero. Bogotá: Decisiones Informadas en Medicamentos; 2020[acceso 16 jun 2020]. Disponible en http://www.proyectodime.info/informacion-regional/ informacion-seleccionada-sobre-COVID-19/

8. Universidad Pablo de Olavide. Biblioteca/CRAI. La Biblioteca/CRAI durante la COVID-19: informaciones básicas y recursos de información durante la crisis del coronavirus. Guias y tutoriales de la Biblioteca/CRAI. 2020[acceso 16 jun 2020]. Disponible en: https://guiasbib.upo.es/ biblioteca_covid_19/articulos

9. Ministerio de Ciencia e Innovación (ES). Guía COVID19: información para profesionales sanitarios. Madrid: Ministerio de Ciencia e Innovación; 2020[acceso 16 jun 2020]. Disponible en: https: / /www.isciii.es/QueHacemos/ Servicios/Biblioteca/Paginas/Gu\%C3\%ADa-COVID-19.Informaci\%C3\%B3n-para-profesionales-sanitarios.aspx

10. US National Library of Medicine - NLM. Clinical trials. Washington: US National Library of Medicine; 2020[acceso 16 jun 2020]. Disponible en: https://clinicaltrials.gov/ct2/results?recrs=ab\&cond=COVID$19 \&$ term=\&cntry=US\&state=\&city=\&dist=

11. US National Library of Medicine - NLM. Randomised evaluation of COVID-19 therapy (RECOVERY). Clinical trials. 3 jun 2020[acceso 3 jun 2020]. Disponible en: https://clinicaltrials.gov/ct2/show/NCT04381936

12. Wang $M$, Cao R, Zhang L, Yang X, Liu J, Xu M et al. Remdesivir and chloroquine effectively inhibit the recently emerged novel coronavirus
(2019-nCoV) in vitro. Cell Res. 2020;30(3):269-71. https://doi.org/10.1038/s41422-020-0282-0

13. Yao $X$, Ye F, Zhang $M$, Cui $C$, Huang $B$, Niu $P$ et al. In vitro antiviral activity and projection of optimized dosing design of hydroxychloroquine for the treatment of severe acute respiratory syndrome coronavirus 2 (SARS-COV-2). Clin Infect Dis. 2020;71(15):732-9. https://doi.org/10.1093/cid/ciaa237

14. McIntosh K. Coronavirus disease 2019 (COVID-19). Wolters Kluwer Uptodate. 13 mar 2020[acceso 23 mar 2020]. Disponible en: https://www.cmim.org/PDF_covid/ Coronavirus_disease2019_COVID-19_UpToDate2.pdf

15. The Multicenter Collaboration Group of Department of Science and Technology of Guangdong Province. Expert consensus on chloroquine phosphate for the treatment of novel coronavirus pneumonia. Chin J Tuberc Respir Dis. 2020;43(3):185-8. https://doi.org/10.3760/cma.j.issn.1001-0939.2020.03.009

16. Chen J, Liu D, Liu L, Liu P, Xu Q, Xia L et al. A pilot study of hydroxychloroquine in treatment of patients with common coronavirus disease-19 (COVID-19). Zhejiang Da Xue Xue Bao Yi Xue Ban. 2020;49(2):215-9. https://doi.org/10.3785/j.issn.1008-9292.2020.03.03

17. Chen Z, Hu J, Zhang Z, Jiang S, Han S, Yan D et al. Efficacy of hydroxychloroquine in patients with COVID-19: results of a randomized clinical trial. MedRvix. 2020:1-11. https://doi.org/10.1101/2020.03.22.20040758

18. Tang W, Cao Z, Han M, Wang Z, Chen J, Sun W et al. Hydroxychloroquine in patients with mainly mild to moderate coronavirus disease 2019: open label, randomised controlled trial. BMJ 2020;369:1-11. https: / / doi.org/10.1136/bmj.m1849

19. Borba MGS, Val FFA, Sampaio VS, Alexandre MAA, Melo GS, Brito M et al. Effect of high vs low doses of chloroquine diphosphate as adjunctive therapy for patients hospitalized with severe acute respiratory syndrome coronavirus 2 (SARS-COV-2) infection: a randomized clinical trial. JAMA Netw Open. 2020;3(4):1-14. https://doi.org/10.1001/jamanetworkopen.2020.8857

20. Boulware DR, Pullen MF, Bangdiwala AS, Pastick KA, Lofgren $\mathrm{SM}$, Okafor EC et al. A randomized trial of hydroxychloroquine as postexposure prophylaxis for COVID-19. N Engl J Med. 2020;383:517-25. https://doi.org/10.1056/NEJMoa2016638

21. Chen L, Zhang ZY, Fu JG, Feng ZP, Zhang SZ, Han QY et al. Efficacy and safety of chloroquine or hydroxychloroquine in moderate type of COVID-19: a prospective openlabel randomized controlled study. MedRxiv. 2020:1-27. https: / / doi.org/10.1101/2020.06.19.20136093

22. Horby P, Mafham M, Linsell L, Bell JL, Staplin N, Emberson $J R$ el at. Effect of hydroxychloroquine in hospitalized patients with COVID-19: preliminary results from a multicentre, randomized, controlled trial. MedRxiv 2020:1-27. https://doi.org/10.1101/2020.07.15.20151852

23. Skipper CP, Pastick KA, Engen NW, Bangdiwala AS, Abassi $M$, Lofgren SM et al. Hydroxychloroquine in nonhospitalized adults with early COVID-19: a randomized trial. Ann Intern Med. 2020. https://doi.org/10.7326/M20-4207 
24. Gautret P, Lagier J-C, Parola P, Hoang VT, Meddeb L, Mailhe $M$ et al. Hydroxychloroquine and azithromycin as a treatment of COVID-19: results of an open-label non-randomized clinical trial. Int J Antimicrob Agents. 2020;56(1):1-6. https://doi.org/10.1016/j.ijantimicag.2020.105949

25. Rosenberg ES, Dufort EM, Udo T, Wilberschied LA, Kumar J, Tesoriero J et al. Association of treatment with hydroxychloroquine or azithromycin with in-hospital mortality in patients with COVID-19 in New York state. JAMA. 2020;323(24):2493-502. https://doi.org/10.1001/jama.2020.8630

26. Geleris J, Sun Y, Platt J, Zucker J, Baldwin M, Hripcsak $G$ et al. Observational study of hydroxychloroquine in hospitalized patients with COVID-19. N Engl J Med. 2020;382:2411-8. https://doi.org/10.1056/NEJMoa2012410

27. Magagnoli J, Narendran S, Pereira F, Cummings T, Hardin JW, Sutton SS et al. Outcomes of hydroxychloroquine usage in United States veterans hospitalized with COVID-19. MedRvix. 2020:1-27. https://doi.org/10.1101/2020.04.16.20065920

28. Mahévas $M$, Tran VT, Roumier $M$, Chabrol A, Paule R, Guillaud $C$ et al. Clinical efficacy of hydroxychloroquine in patients with COVID-19 pneumonia who require oxygen: observational comparative study using routine care data. BMJ. 2020;369:1-9. https://doi.org/10.1136/bmj.m1844

29. Gautret P, Lagier JC, Parola P, Hoang VT, Meddeb L, Sevestre $\mathrm{J}$ et al. Clinical and microbiological effect of a combination of hydroxychloroquine and azithromycin in 80 COVID-19 patients with at least a six-day follow up: a pilot observational study. Travel Med Infect Dis. 2020;34:1-7. https://doi.org/10.1016/j.tmaid.2020.101663

30. Million M, Lagier JC, Gautret P, Colson P, Fournier P-E, Amrane $S$ et al. Early treatment of COVID-19 patients with hydroxychloroquine and azithromycin: a retrospective analysis of 1.061 cases in Marseille, France. Travel Med Infect Dis. 2020;35:1-9. https://doi.org/10.1016/j.tmaid.2020.101738

31. Molina JM, Delaugerre C, Goff J, Mela-Lima D, Ponscarme $D$, Goldwirt $L$ et al. No evidence of rapid antiviral clearance or clinical benefit with the combination of hydroxychloroquine and azithromycin in patients with severe COVID-19 infection. Med Mal Infect. 2020;50(4):3827. https://doi.org/10.1016/j.medmal.2020.03.006

32. Red Argentina de Centros de Información de Medicamentos - RACIM. Ficha técnica RACIM: hidroxicloroquina, sulfato de. Córdoba: Centros de Información de Medicamentos; 2020[acceso 3 mayo 2020]. Disponible en: http://cime.fcq. unc.edu.ar/wp-content/uploads/sites/15/2020/05/RACIMHidroxicloroquina-versi\%C3\%B3n1-03.05.2020.pdf

33. Caffaratti M. Precaución con el uso de hidroxicloroquina en pacientes con enfermedad cardiovascular y COVID19. Córdoba: Centro de Información de Medicamentos; 2020[acceso 25 abr 2020]. Disponible en: http:cime. fcq.unc.edu.ar/wp-content/uploads/sites/15/2020/04/ Informe-CIME-uso-HCQ-v.final.pdf

34. Agencia Española de Medicamentos y Productos Sanitarios - AEMPS. Cloroquina e hidroxicloroquina: precauciones y vigilancia de posibles reacciones adversas em pacientes con COVID-19. Madrid: Agencia Española de Medicamentos y Productos Sanitarios; 2020[acceso 16 mayo 2020]. Disponible en: http://www.aemps.gob.es/informa/ notasinformativas/medicamentosusohumano-3/seguridad1/2020-seguridad-1/cloroquina-hidroxicloroquinaprecauciones-y-vigilancia-de-posibles-reacciones-adversasen-pacientes-con-COVID-19

35. Wang Y, Zhang D, Du G, Du R, Zhao J, Jin Y et al. Remdesivir in adults with severe COVID-19: a randomised, double-blind, placebo-controlled, multicentre trial. Lancet. 2020;395(10236):1569-78. https://doi.org/10.1016/S0140-6736(20)31022-9

36. Beigel JH, Tomashek KM, Dodd LE, Mehta AK, Zigman BS, Kalil AC. Remdesivir for the treatment of COVID19: preliminary report. New Engl J Med. 2020:1-12. https://doi.org/10.1056/NEJMoa2007764

37. Grein J, Ohmagari N, Shin D, Díaz G, Asperges E, Castagna A et al. Compassionate use of remdesivir for patients with severe COVID-19. N Engl J Med. 2020;382:2327-36. https://doi.org/10.1056/NEJMoa2007016

38. Antinori S, Cossu M, Ridolfo A, Rech R, Bonazzetti C, Pagani $G$ et al. Compassionate remdesivir treatment of severe COVID-19 pneumonia in intensive care unit (ICU) and non-ICU patients: clinical outcome and differences in post treatment hospitalisation status. Pharmacol Res. 2020;158:1-6. https://doi.org/10.1016/j.phrs.2020.104899

39. Goldman JD, Lye DCB, Hui DS, Marks KM, Bruno R, Montejano $\mathrm{R}$ et al. Remdesivir for 5 or 10 days in patients with severe COVID-19. N Engl J Med. 2020:1-11. https://doi.org/10.1056/NEJMoa2015301

40. US Food and Drug Administration - FDA. Fact sheet for health care providers: emergency use authorization (EUA) of remdesivir (GS-5734). Washington: US Food and Drug Administration; 2020[acceso 1 mayo 2020]. Disponible en: https://www.fda.gov/media/137566/download

41. Fundación Instituto Catalán de Farmacología - FICF. Sistema de información esencial en terapéutica y salud (Sietes): Ref ID 103634. Barcelona: Fundación Instituto Catalán de Farmacología; 2020[acceso 27 mayo 2020]. Disponible en: http://www.sietes.org/buscar/cita/103634

42. Sheahan TP, Sims AC, Leist SR, Schäfer A, Won J, Brown AJ et al. Comparative therapeutic efficacy of remdesivir and combination lopinavir, ritonavir, and interferon beta against MERS-CoV. Nat Commun. 2020;11(1):1-14. https://doi.org/10.1038/s41467-019-13940-6

43. Cao B, Wang Y, Wen D, Liu W, Wang J, Fan G et al. A trial of lopinavir-ritonavir in adults hospitalized with severe COVID-19. N Engl J Med. 2020;382:1787-99. https://doi.org/10.1056/NEJMoa2001282

44. Hung IFN, Lung KC, Tso EYK, Liu R, Chung TWH, Chu MY et al. Triple combination of interferon beta- $1 \mathrm{~b}$, lopinavirritonavir, and ribavirin in the treatment of patients admitted to hospital with COVID-19: an open-label, randomised, phase 2 trial. Lancet. 2020;395(10238):1695-70. https://doi.org/10.1016/S0140-6736(20)31042-4 
45. Randomised Evaluation of COVID-19 Therapy - RECOVERY. Statement from the chief investigators of the randomised evaluation of COVID-19 therapy (RECOVERY) trial on lopinavir-ritonavir. London: University of Oxford; 2020[acceso 29 jun 2020]. Disponible en: https: / /www. recoverytrial.net/files/lopinavir-ritonavir-recoverystatement-29062020_final.pdf

46. Young BE, Ong SWX, Kalimuddin S, Low JG, Tan SY, Loh J et al. Epidemiologic features and clinical course of patients infected with SARSCoV-2 in Singapore. JAMA. 2020:323(15):1488-94. https://doi.org/10.1001/jama.2020.3204

47. Agencia Española de Medicamentos y Productos Sanitarios - AEMPS. Tratamientos disponibles para el manejo de la infección respiratoria por SARS-CoV-2. Madrid: Agencia Española de Medicamentos y Productos Sanitarios; 2020[acceso 9 jul 2020]. Disponible en: https: / /www. aemps.gob.es/la-aemps/ultima-informacion-de-la-aempsacerca-del-covid\%E2\%80\%9119/tratamientos-disponiblespara-el-manejo-de-la-infeccion-respiratoria-por-sars-cov-2/

48. Villar J, Ferrando C, Martínez D, Ambrós A, Muñoz T, Soler JA et al. Dexamethasone treatment for the acute respiratory distress syndrome: a multicentre, randomised controlled trial. Lancet Respir Med. 2020;8(3):267-76. https://doi.org/10.1016/S2213-2600(19)30417-5

49. Randomised Evaluation of COVID-19 Therapy - RECOVERY. Dexamethasone in hospitalized patients with COVID19: preliminary report. New Engl J Med. 2020:1-11. https://doi.org/10.1056/NEJMoa2021436

50. Ye Z, Wang Y,Colunga-Lozano LE, Prasad $M$, Tangamornsuksan $W$ et al. Efficacy and safety of corticosteroids in COVID-19 based on evidence for COVID-19, other coronavirus infections, influenza, communityacquired pneumonia and acute respiratory distress syndrome: a systematic review and meta-analysis. CMAJ. 2020;192(27):E756-67. https://doi.org/10.1503/cmaj.200645

51. Nicholls JM, Poon LLM, Lee KC, Ng WF, Lai ST, Leung $\mathrm{CY}$ et al. Lung pathology of fatal severe acute respiratory syndrome. Lancet. 2003;361(9371):1773-8. https://doi.org/10.1016/S0140-6736(03)13413-7

52. Nicholls J, Dong XP, Jiang G, Peiris M. SARS: clinical virology and pathogenesis. Respirology. 2003;8(supl 1):s6-s8. https://doi.org/10.1046/j.1440-1843.2003.00517.x

53. Siddiqi HK, Mehra MR. COVID-19 illness in native and immunosuppressed states: a clinical-therapeutic staging proposal. J Hearth Lung Transplant. 2020;39(5):405-7. https://doi.org/10.1016/j.healun.2020.03.012

54. Mehra MR, Desai SS, Ruschitzka F, Patel AN. Retracted: hydroxychloroquine or chloroquine with or without a macrolide for treatment of COVID-19: a multinational registry analysis. Lancet. 2020:1-10. https://doi.org/10.1016/S0140-6736(20)31180-6

55. Mak TK, Lim JCW, Thanapholler P, Mahlangu GN, Cooke $\mathrm{E}$, Lumpkin MM. Global regulatory agility during COVID19 and other health emergencies. BMJ. 2020;369:1-2. https://doi.org/10.1136/bmj.m1575 pmid:32340997

56. Durán C, Cañás M, Chistiaens T. EMA and FDA decisions based on flawed evidence to approve new cancer drugs negatively affect Latin American patients. BMJ. 2019;367. https://doi.org/10.1136/bmj.16017

57. Cañás M, Urtasun MA. La evidencia en tiempos de coronavirus (COVID-19). Evid Actual Pract Ambul. 2020;23(2):1-5.

58. Fernández LCS. Cloroquina e hidroxicloroquina como potenciales terapias frente al COVID-19. Madrid: Centro Cochrane Iberoamericano; 2020[acceso 5 jun 2020]. Disponible en: https://es.cochrane.org/sites/es.cochrane. org/files/public/uploads/COVID-19/cochrane_navarra_ COVID-19_cloroquina_hidroxicloroquina.pdf

59. Cañás M, Urtasun MA. Entre la ilusión y la ciencia. Revista Mestiza. 2020[acceso 27 abr 2020]. Disponible en: https:// revistamestiza.unaj.edu.ar/entre-la-ilusion-y-la-ciencia/

60. Ferner RE, Aronson JK. Remdesivir in COVID-19: a drug with potential: dont' $t$ waste time on uncontrolled observations. BMJ. 2020;369:1-2. https://doi.org/10.1136/bmj.01610

\section{Contribuição dos Autores}

Saavedra PAE, Cañás M, Barbado DMC, Esparza LB, Caffaratti M, Speranza N, Martínez CF, Gutiérrez JJL- Concepção, planejamento (desenho do estudo), aquisição, análise, interpretação dos dados e redação do trabalho. Todos os autores aprovaram a versão final do trabalho.

\section{Conflito de Interesse}

Os autores informam não haver qualquer potencial conflito de interesse com pares e instituições, políticos ou financeiros deste estudo.

Esta publicação está sob a licença Creative Commons Atribuição 3.0 não Adaptada.

Para ver uma cópia desta licença, visite http://creativecommons.org/licenses/by/3.0/deed.pt_BR. 